\title{
Nitric oxide is involved in the brassinolide- induced adventitious root development in cucumber
}

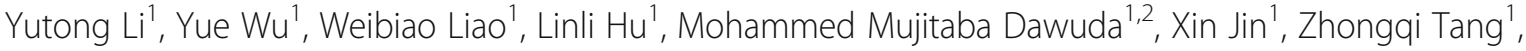 \\ Jianjun Yang ${ }^{1}$ and Jihua $\mathrm{Yu}^{1 *}$ (D)
}

\begin{abstract}
Background: Brassinolide (BR), as a new type of plant hormones, is involved in the processes of plant growth and stress response. Previous studies have reported the roles of BR in regulating plant developmental processes and also response tolerance to abiotic stresses in plants. The main purpose of our study was to explore whether nitric oxide (NO) plays a role in the process of BR-induced adventitious root formation in cucumber (Cucumis sativus L.).

Results: Exogenous application of $1 \mu \mathrm{M}$ BR significantly promoted adventitious rooting, while high concentrations of BR (2-8 $\mu \mathrm{M})$ effectively inhibited adventitious rooting. NO donor (S-nitroso-N-acerylpenicillamine, SNAP) promoted the occurrence of adventitious roots. Simultaneously, BR and SNAP applied together significantly promoted adventitious rooting and the combined effect was superior to the application of BR or SNAP alone. Moreover, NO scavenger (C-PTIO) and inhibitors (L-NAME and Tungstate) inhibited the positive effects of BR on adventitious rooting. BR at $1 \mu \mathrm{M}$ also increased endogenous NO content, NO synthase (NOS-like) and Nitrate reductase (NR) activities, while BRz (a specific BR biosynthesis inhibitor) decreased these effects. In addition, the relative expression level of $N R$ was up-regulated by BR and SNAP, whereas BRz down-regulated it. The application of NO inhibitor (Tungstate) in BR also inhibited the up-regulation of NR.
\end{abstract}

Conclusion: BR promoted the formation of adventitious roots by inducing the production of endogenous NO in cucumber.

Keywords: Brassinolide, Nitric oxide, Nitric oxide synthase, Nitrate reductase, Nitrate reductase genes

\section{Background}

Brassinolide (BR), a new plant hormone, was first discovered during the screening of pollen grains [1]. As a steroid hormone, it plays an important role in regulating various developmental processes, including root and hypocotyl elongation [2] . Moreover, it mediates plant responses to various stimuli, such as hypoxia stress [3], chilling injury [4], salt stress [5], heavy metal stress [6] and drought stress

\footnotetext{
* Correspondence: yujihuagg@163.com

${ }^{1}$ College of Horticulture, Gansu Agricultural University, Lanzhou 730070, People's Republic of China

Full list of author information is available at the end of the article
}

[7]. Yuan et al. [8] reported that the application of 2,4-Epibrassinolide (EBL) alleviated $\mathrm{Ca}\left(\mathrm{NO}_{3}\right)_{2}$ stress in cucumber plants by regulating mineral nutrients uptake and distribution. Zhao et al. [9] also found that exogenous EBR application ameliorated the inhibitory effects of photosynthesis, antioxidant enzyme activity and Rubisco activase (RCA) gene expression in Triticum aestivum induced by a combination of drought and heat stress. The effect of BR on plant growth and development processes depends on the concentration. Low concentration of BR was suitable for callus growth and shoot regeneration in Spartina patens [10], while high concentration of epibrassinolide inhibited

(c) The Author(s). 2020 Open Access This article is licensed under a Creative Commons Attribution 4.0 International License, which permits use, sharing, adaptation, distribution and reproduction in any medium or format, as long as you give appropriate credit to the original author(s) and the source, provide a link to the Creative Commons licence, and indicate if changes were made. The images or other third party material in this article are included in the article's Creative Commons licence, unless indicated otherwise in a credit line to the material. If material is not included in the article's Creative Commons licence and your intended use is not permitted by statutory regulation or exceeds the permitted use, you will need to obtain permission directly from the copyright holder. To view a copy of this licence, visit http://creativecommons.org/licenses/by/4.0/ The Creative Commons Public Domain Dedication waiver (http://creativecommons.org/publicdomain/zero/1.0/) applies to the data made available in this article, unless otherwise stated in a credit line to the data. 
the growth of Brassica oleraceae cotyledons [11]. Brassinazole $\left(\mathrm{BR}_{\mathrm{Z}}\right)$ is a specific $\mathrm{BR}$ biosynthesis inhibitor. $\mathrm{BR}_{\mathrm{Z}}$ treated cress showed dwarfism, with altered leaf morphology, including the downward curling and dark green color typical of Arabidopsis BR-deficient mutants and the application of $10 \mathrm{nM}$ brassinolide could reverse the dwarfism [12].

Nitric oxide (NO), a ubiquitous signal molecule, plays important roles in different plant tissues and participates in a variety of physiological processes [13]. Many researchers observed that NO induced root development in Zea mays [14], and it also induced seed germination, seedling development, stomatal responses, senescence, flowering and protection against pathogens in different plant species [15-20]. NO production in plants has two pathways, including enzymatic pathway and non-enzymatic pathway. Nitrate reductase (NR) and NO synthase (NOS)like enzyme are the NO-producing enzymes identified in plants [21]. Zhu et al. [22] have reported that NO production through NOS and NR pathways was involved in adventitious rooting of cucumber explants induced by $\mathrm{H}_{2}$. The activities of NR and NOS-like enzymes were involved in BR signaling [23]. Moreover, as the second messenger, NO could interact with some hormones to regulate plant physiological and biochemical responses. It is involved in the signaling pathways of salicylic acid (SA), cytokinin (CTK), jasmonic acid (JA), ethylene (ETH), hydrogen peroxide $\left(\mathrm{H}_{2} \mathrm{O}_{2}\right)$ and indole-3-acetic acid (IAA) [24-28]. Pagnussat et al. [29] reported the role of IAA and NO in the signaling pathway during the effect of exogenous IAA on the adventitious roots of cucumber. It was clarified that NO operates downstream of IAA promoting adventitious root development through the GC-catalyzed synthesis of cGMP. Both $\mathrm{NO}$ and $\mathrm{H}_{2} \mathrm{O}_{2}$ played crucial roles and had synergistic effect on adventitious root development in marigold (Tagetes erecta L.) [30].

The formation of adventitious roots is a fundamental process of root biology, through which cells of adventitious roots form new roots after the embryo. The development of adventitious roots is a complex process regulated by various environment and plants hormones factors [31, 32]. Pagnussat et al. [27] observed that a transient increase in NO concentration was required and was part of the molecular events involved in adventitious root development induced by indole acetic acid (IAA), indicating that NO mediates the auxin response leading the adventitious root formation. BR-enhanced water stress tolerance in maize plants was due to BR-induced NO production and NOactivated ABA biosynthesis [33]. The existence of a signaling pathway leading to BR-mediated systemic virus resistance involves local Respiratory Burst Oxidase Homolog B ( $\mathrm{RBOHB}$ )-dependent $\mathrm{H}_{2} \mathrm{O}_{2}$ production and subsequent systemic NR-dependent NO generation [34]. Kwak et al. [35] reported that lower concentrations of $\mathrm{BL}$ increased the number and length of adventitious roots while higher concentrations of BL caused trichome-like roots. As mentioned above, both BR and NO could promote adventitious root development, which suggest a possible relationship between BR and NO. Karpets and Kolupaev [36] reported that NO was involved in 2,4-epibrassinolideinduced heat resistance of wheat coleoptiles and the functional interaction between NO, ROS, and calcium ions as the signal mediators. Until now, many researches focused on studying the relationship between $\mathrm{NO}$ and other plant hormones [24-27]. However, little is known about the relationship between BR and NO during the development of adventitious roots. To explore this issue, pharmacological experiments were conducted using cucumber (Cucumis sativus L.) as test material to investigate the role of $\mathrm{NO}$ in BR-induced adventitious roots development. The results provide new insights into the involvement of $\mathrm{NO}$ in BRinduced adventitious roots development in cucumber.

\section{Result \\ BR concentrations affect number and length of adventitious roots}

In order to investigate the effect of BR on adventitious roots, the cucumber explants were exposed to different concentrations of $\mathrm{BR}(0,0.2,1,2,4$ and $8 \mu \mathrm{M})$. The root length and root number of adventitious roots initially increased and then decreased with the increase of BR concentration, both reaching the maximum values at $1 \mu \mathrm{M}$ (Fig. 1). Thus, the optimum concentration of BR $(1 \mu \mathrm{M})$ was used in the subsequent experiment.

\section{Number and length of adventitious root are affected by NO scavenger and inhibitors}

The effect of NO scavenger (c-PTIO), NOS-like enzyme inhibitor (L-NAME) and NR inhibitor (Tungstate) on BR-induced adventitious rooting was investigated. As shown in Fig. 2, compared with BR treatment, $200 \mu \mathrm{M}$ c-PTIO, $20 \mu \mathrm{M}$ L-NAME or $100 \mu \mathrm{M}$ Tungstate applied in combination with BR treatment significantly inhibited adventitious root formation. The adventitious root number and length of explants treated with SNAP (NO donor) plus BR were significantly higher than those of explants treated with either BR or SNAP alone.

\section{Temporal regulation of endogenous NO content, NOS-like and NR activity by BR}

The time-course of NO content as affected by $1 \mu \mathrm{M}$ BR or $1.5 \mu \mathrm{M} \mathrm{BR} \mathrm{Z}_{\mathrm{Z}}$ treatment is shown in Fig. 3a. Compared with the control, the NO content of BR-treated explants has a slow downward trend at $0 \mathrm{~h}-6 \mathrm{~h}$, which may be due to the wound response. From $6 \mathrm{~h}$ to $24 \mathrm{~h}$, the NO content of BRtreated explants increased, and subsequently gradually decreased until $48 \mathrm{~h}$ (Fig. 3a). The content of NO in the BR treatment reached the maximum at $24 \mathrm{~h}$ and was about 

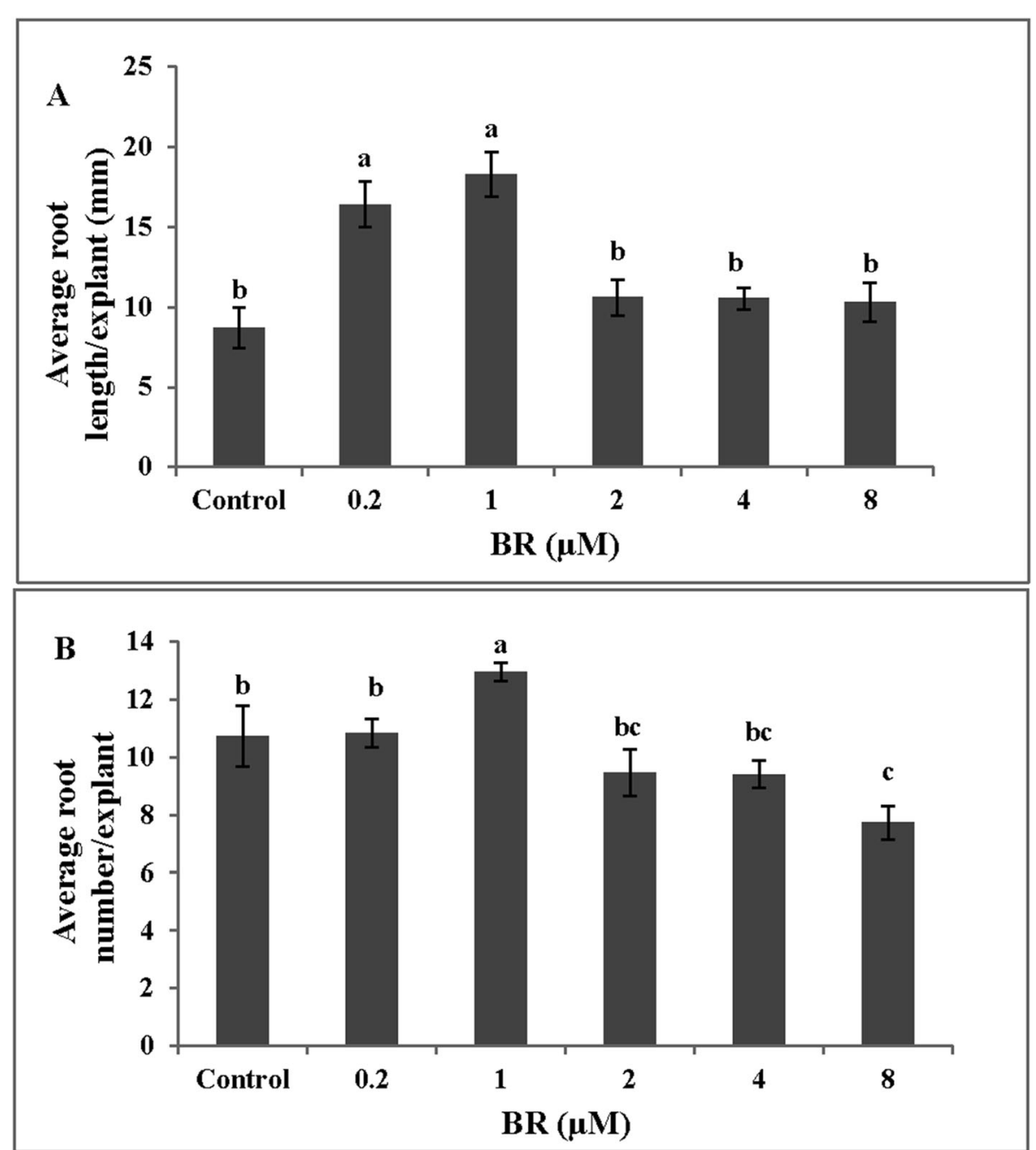

Fig. 1 Effect of different concentrations of BR on the average root length and the average root number of cucumber adventitious roots development. Photographs were taken after 5 days of treatment. The different concentration of BR adventitious root length (a) and root numbers (b) were expressed as means \pm SE ( $n=10$ explants from three independent experiments). Bars denoted by different letters were significantly different according to Duncan's multiple test $(P<0.05)$

1.8 times as compared to the control. In addition, the NO content of BRz-treated explants gradually decreased from $0 \mathrm{~h}$ to $48 \mathrm{~h}$, and the NO levels was always lower than those of BR-treated explant. Thus, the data suggest that BR regulated endogenous NO to promote the development of adventitious roots in cucumber.

To explore the relationship between BR and NO, we further analyzed the effects of BR on the activities of NOS-like and NR enzymes in cucumber explants during the formation of adventitious roots (Fig. $3 \mathrm{~b}$ and c). The application of BR distinctly affected the time course of NOS-like activity. The NOS-like activity in BR-treated explants decreased slightly at $0-6 \mathrm{~h}$, and then increased from $6 \mathrm{~h}$ to $24 \mathrm{~h}$, reaching a maximum at $24 \mathrm{~h}$, which was about 2-fold of the control. Then, from $24 \mathrm{~h}$ to $48 \mathrm{~h}$, NOS-like activity tended to gradually decreased (Fig. 3b). Meanwhile, compared with the control and the BR treatment, the NOS-like enzyme activity of $\mathrm{BR}_{\mathrm{Z}}$ treatment consistently decreased throughout the whole experiment (Fig. 3b). Similarly, the NR activity of explants treated with BR decreased transiently during the first $6 \mathrm{~h}$ period, followed by a significant increase from $6 \mathrm{~h}$ to 24 $\mathrm{h}$, which reached its highest activities at $24 \mathrm{~h}$ (about 1.4fold of the control), and then decreased at $48 \mathrm{~h}$ (Fig. 3c). However, the NR activity in BRz-treated explants continuously decreased during the time of experiment (Fig. $3 c)$. In conclusion, the activity of NOS-like and NR enzymes were promoted by BR treatment, while the $\mathrm{BRz}$ inhibited the activities of these two enzymes. Here, we showed that BR regulated the production of endogenous NO by inducing the increase activity of NOS-like and NR enzyme during the adventitious root formation. 

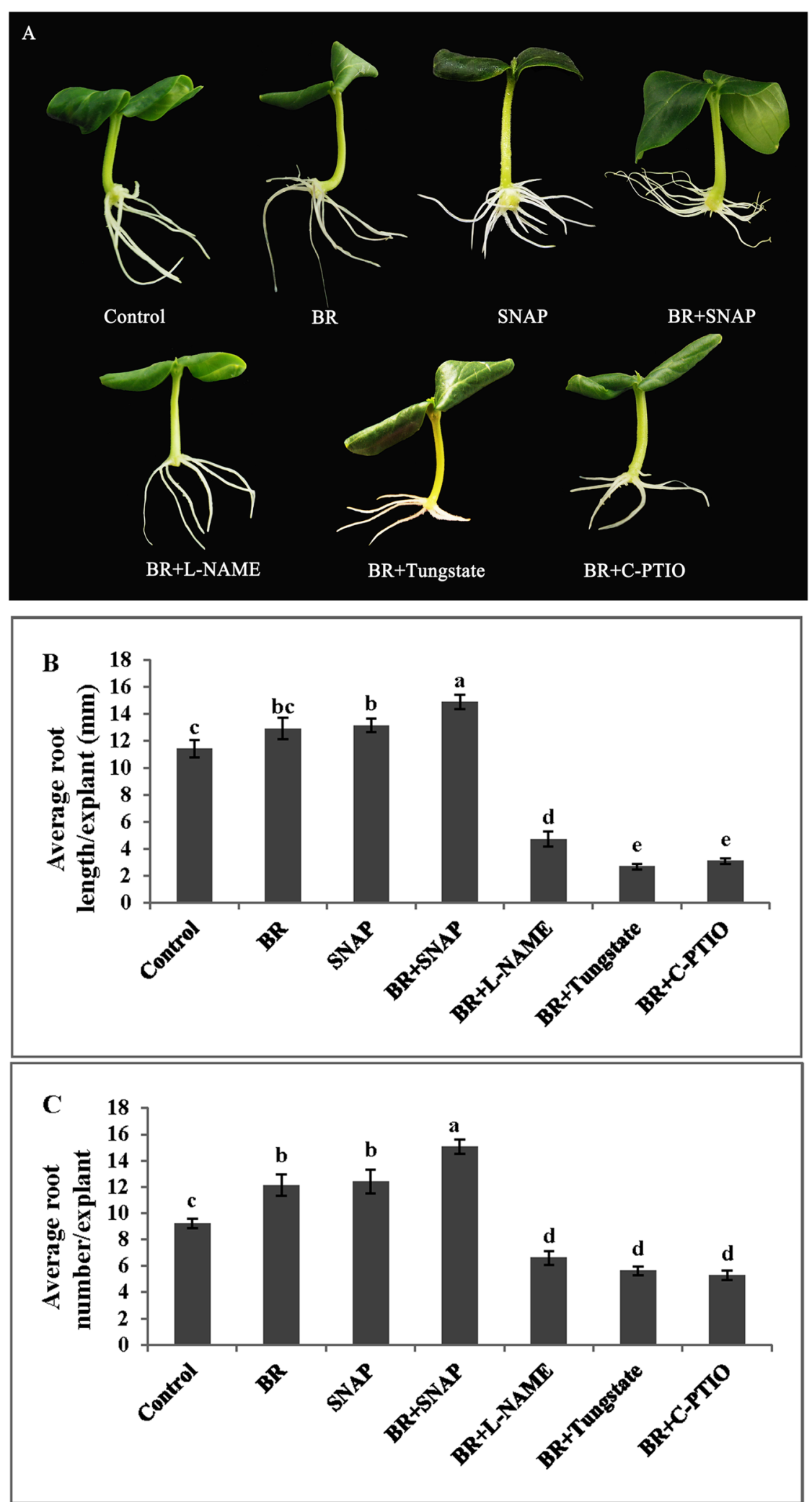

Fig. 2 (See legend on next page.) 
(See figure on previous page.)

Fig. 2 Effect of NO scavenger or inhibitors on BR-induced adventitious root formation. The primary root was removed from hypocotyls of 5-dayold germinated cucumber seedling. Explants were incubated in distilled water, $1 \mu \mathrm{M}$ BR, $50 \mu \mathrm{M}$ SNAP, $20 \mu \mathrm{M} \mathrm{L-NAME}, 100 \mu \mathrm{M}$ Tungstate, and $200 \mu \mathrm{M}$ c-PTIO for 5 days. Photographs were taken 5 days after treatment (a). Adventitious root length (b) and root numbers (c) were expressed as means \pm SE ( $n=10$ explants from three independent experiment). Bars denoted by different letters were significantly different according to Duncan's multiple test $(P<0.05)$

NO content, NOS-like and NR activity under BR, SNAP, L$\mathrm{NAME}$, tungstate and $\mathrm{BR}_{\mathrm{Z}}$ treatments

In order to further verify whether $\mathrm{NO}$ participates in BR-induced adventitious roots formation in cucumber, the explants were placed in BR, SNAP, BR + L-NAME, $\mathrm{BR}+$ Tungstate and $\mathrm{BRz}$ treatment for $24 \mathrm{~h}$. The fluorescence localization of NO in hypocotyl, the content of endogenous NO and the activities of NR and NOS-like enzymes were analyzed. As shown in Fig. $4 \mathrm{a}$ and b, after treatment with BR and SNAP, brighter green fluorescence was observed in the tissue at the place where hypocotyl produce adventitious roots, and the intensity of fluorescence was significantly higher than in control explants, indicating that the production of $\mathrm{NO}$ was sharply rising. In opposite, explants treated with $\mathrm{BR}+\mathrm{L}-$ NAME, BR+ Tungstate and BRz showed a lower fluorescence in the hypocotyl than in the control plants (Fig. 4a and b). In order to support the qualitative analysis, the quantification of endogenous NO content was done in hypocotyl of cucumber explants. As shown in Figs. 4c, compared with the control, endogenous NO content after treatment with BR and SNAP was significantly increased by 78.03 and $84.79 \%$, respectively. Compared with the BR treatment, when L-NAME and Tungstate were added to the BR solution, the effects of BR were reversed. Indeed, NO content was reduced by 66.5 and $63.8 \%$, respectively (Fig. $4 \mathrm{c}$ ). Moreover, $\mathrm{BR}_{\mathrm{Z}}$ treatment alone significantly reduced NO content by $68.1 \%$ compared with the BR treatment (Fig. 4c). The qualitative and quantitative analyses of NO in hypocotyl of cucumber explants showed that exogenous application of BR and SNAP significantly increased the production and distribution of endogenous NO in cucumber hypocotyl. As shown in Fig. 4d and e, BR-induced NOS-like and NR activity were blocked by L-NAME and Tungstate. Compared with the control, application of BR and SNAP alone significantly increased the activity of NOS-like enzyme by 40.24 and $45.22 \%$, respectively (Fig. 4d). Moreover, BR + L-NAME and BRz treatments markedly reduced NOS-like enzyme activity by 65.92 and $66.97 \%$ compared with the BR treatment, respectively (Fig. 4d). Similarly, compared with the control, the activity of NR after BR and SNAP treatment was significantly increased by 40.17 and $41.53 \%$, respectively (Fig. 4e). Compared with the BR treatment, the activity of NR enzyme after $\mathrm{BR}+$ Tungstate and $\mathrm{BR}_{\mathrm{Z}}$ treatment was drastically reduced by 41.65 and $43.59 \%$, respectively (Fig. 4e). Thus,
$\mathrm{BR}$ induced the generation of $\mathrm{NO}$ by regulating the activity of NOS-like and NR enzymes, and promoted adventitious root formation in cucumber explants.

\section{The relative expression of $N R$ gene under $B R, B R_{Z}, S N A P$, and tungstate treatments}

During the adventitious rooting process, we performed real time RT-PCR to measure the relative expression of $N R$ gene (Fig. 5). Compared with the control, the NR expression levels in BR- and SNAP- treatment were significantly higher than those in the control at $24 \mathrm{~h}$ after treatment, which were 642.3 and $701.2 \%$ higher over in the control (Fig. 5). There was no significant difference in the relative expression level of $N R$ between $B R+$ tungstate and the control. The relative expression level of $N R$ gene decreased by 89.15 and $89.69 \%$ in BR + Tungstate and $\mathrm{BR}_{\mathrm{Z}}$ treated explants compared with the $\mathrm{BR}$ treatment, respectively (Fig. 5).

\section{Discussion}

The data reported here demonstrated the interaction between BR and NO during adventitious rooting in cucumber explants. The results showed that exogenous BR enhanced the number and length of adventitious root at low concentrations, while high concentrations of BR treatments suppressed adventitious root development (Fig. 1), which suggested that the effect of BR on adventitious root is a dose-dependent response. Previous studies showed that the application of BR $(0.1-10 \mu \mathrm{M})$ promoted hypocotyl elongation in Arabidopsis and the influence was related to the concentrations [2, 38]. Similarly, in tomato, Guan and Roddick [39] reported that the application of 24-epibrassinolide (24-EBR) in excess of $1.0 \mu \mathrm{M}$ reduced root growth as well as root number and root length, but lower concentration of $0.1 \mu \mathrm{M}$ EBR increased the number and root length. It may be noted that the optimum concentration of BR for rooting in previous research $[2,38]$ was different from our experiment. It might be due to the sensitivity to BR of the different plant species.

$\mathrm{BR}$ has biological activity in the bioassay for auxin, which is similar to the function of gibberellin, ethylene and cytokinin, and it affects root formation and development in plants $[40,41]$. BR also participates in the plant life processes, such as responses to various biotic and abiotic stresses [42-44]. Our results also showed that BR promoted adventitious root formation. Furthermore, the 

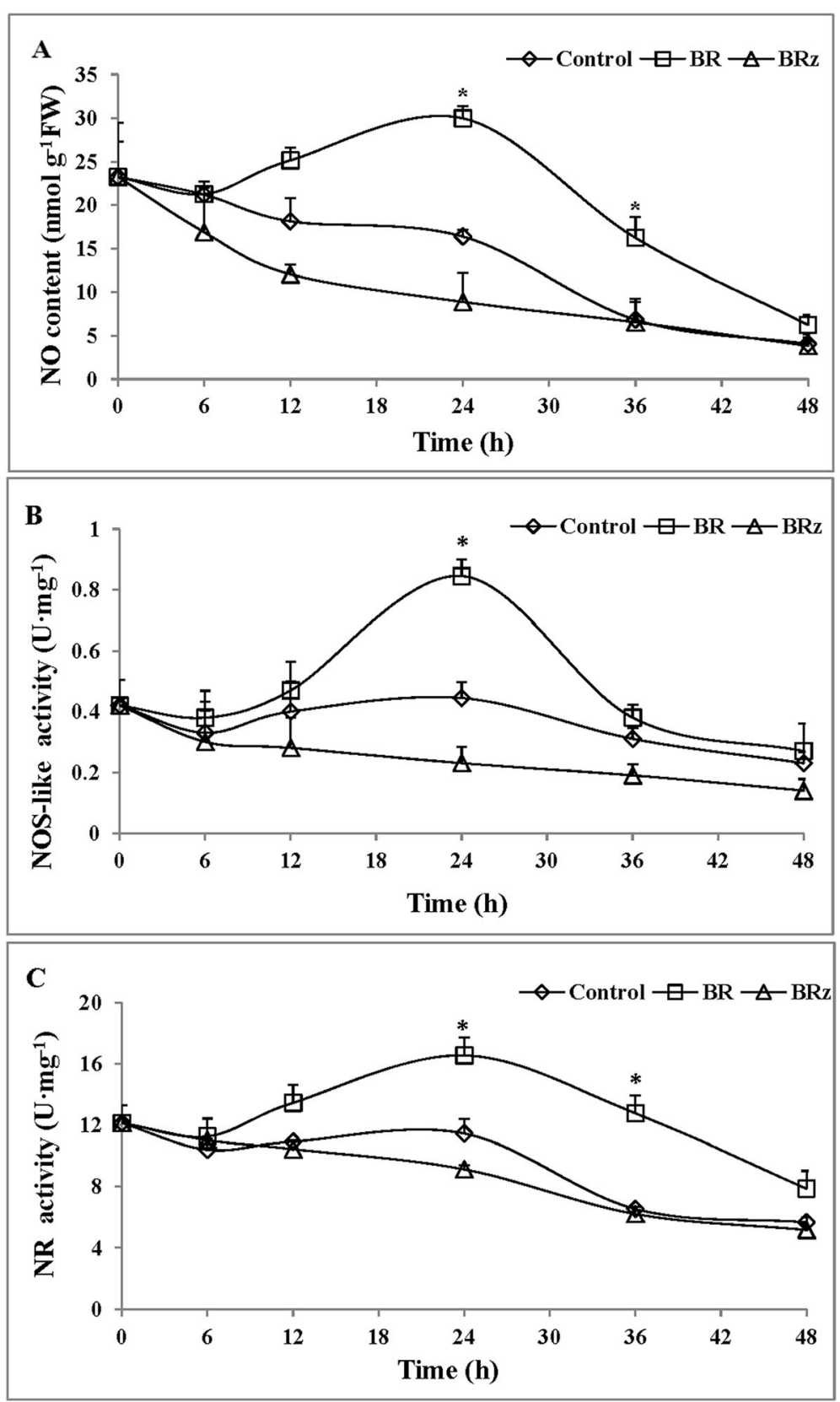

Fig. 3 Effect of BR on endogenous NO content, NOS-like and NR activities in a time- dependent manner. The primary root system was removed from the hypocotyls of 5-d-old germinated cucumber seedlings. Enzymatic activities were measured from $1 \mathrm{~cm}$-long segment taken from the base of hypocotyl. NO levels (a) were determined by Griess reaction reagent in hypocotyls of explants treated with distilled water (control) or $1 \mu \mathrm{M}$ BR, $1.5 \mu \mathrm{M}$ BRz. NOS-like (b) and NR activities (c) of hypocotyls $(1 \mathrm{~cm})$ were determined using the NOS-like and NR determination Kit (Jiancheng, Nanjing, China) according to the manufacturer's instructions in explants treated with distilled water (control) or $1 \mu \mathrm{M}$ BR,1.5 $\mu \mathrm{M}$ BRz. Values (means $\pm \mathrm{SE}$ ) are the averages of three independent experiments ( $n=15$ explants from each of three independent experiments). Asterisks indicate that mean values are significantly different between treatments and control $(P<0.05)$ according to Duncan's multiple test

exogenous application of $\mathrm{BR}_{\mathrm{Z}}$ played a vital role in the inhibition of adventitious root development. The results are consistent with that of Kurepin and co-authors who also found that the application of $\mathrm{BR}_{\mathrm{Z}}$ suppressed hypocotyl growth in Arabidopsis and the effect depended on the concentration applied [2]. In addition, the interaction between BR and other hormones also has a certain effect on the growth and development of plants [45-47]. For example, in Arabidopsis thaliana, BR and auxin signal transduction have interaction points, which induced the synthesis of auxin [48]. The interaction of Brassinolide and ethylene can control the negative gravitropism of 

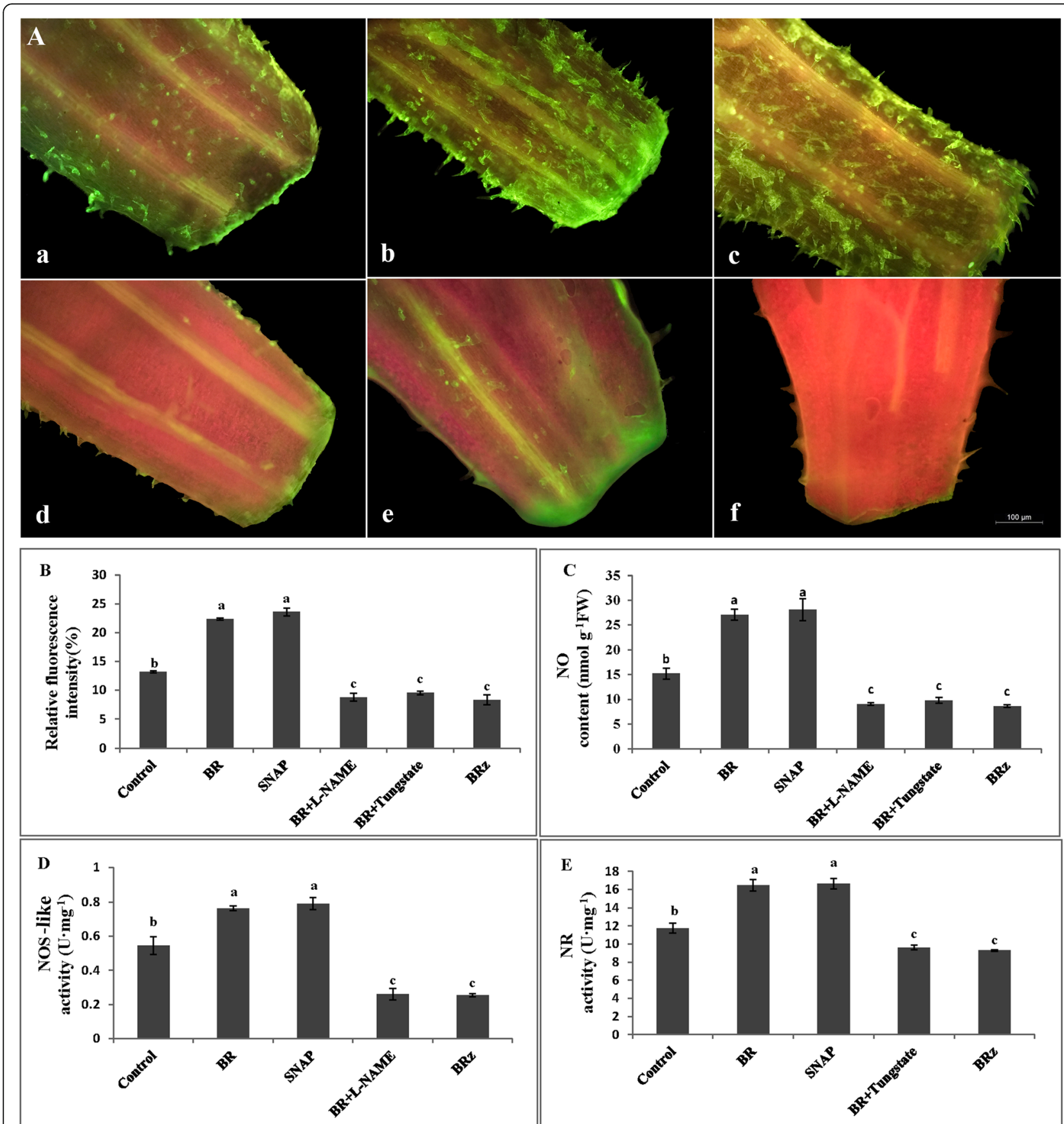

Fig. 4 Effects of BR, SNAP, L-NAME, Tungstate and BRz on the content of NO and BR-induced NOS-like and NR activities. The fluorescence localization

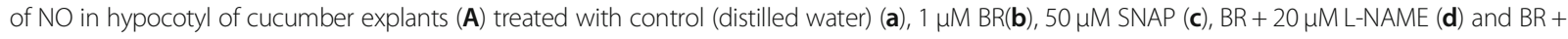
$100 \mu \mathrm{M}$ Tungstate (e), $1.5 \mu \mathrm{M}$ BRz (f) for $24 \mathrm{~h}$. Fluorescence intensity was analyzed using Image J software and expressed as a percentage of the control (B). The quantification of endogenous NO content was done in hypocotyl of cucumber explants (C). NOS-like (D) and NR (E) enzymatic activities were measured from $1 \mathrm{~cm}$-long segment taken from the base of hypocotyl. Mean and SE values were calculated from three independent experiments $(n=$ 15). Bars denoted by the same letter did not differ significantly at $P<0.05$ according to Duncan's multiple test

Arabidopsis seedlings, and it depends on the signal component of auxin [49]. Compared with a single epibrassinolide (BL) treatment, the foliar application of methyl jasmonate (MeJA) and BL in rice resulted in a remarkable reduction in infection of rice black-streaked dwarf virus
(RBSDV) [50]. Moreover, some biological gaseous molecules such as carbon monoxide (CO), $\mathrm{NO}$ and hydrogen peroxide $\left(\mathrm{H}_{2} \mathrm{O}_{2}\right)$ were shown to exhibit similar hormonelike effects as signal transmitters during adventitious rooting [51-53]. It has been confirmed that NO is involved in 


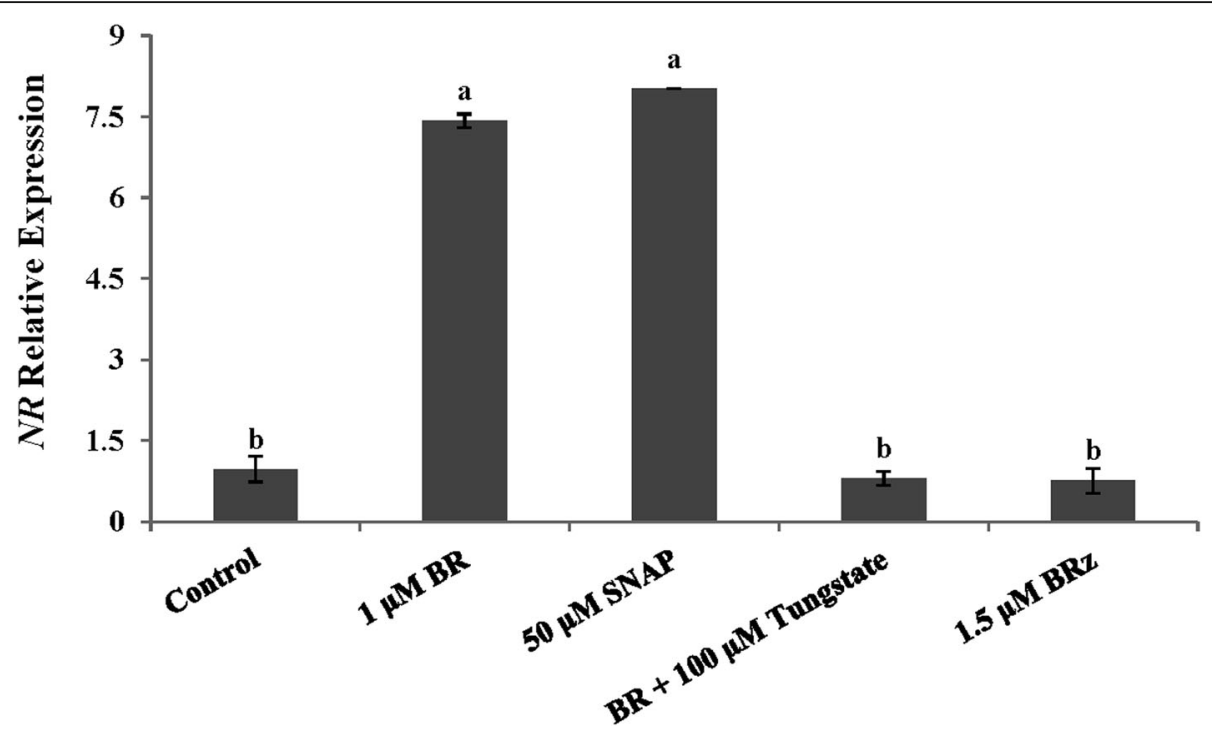

Fig. 5 Analysis by qRT-PCR of the effect of BR, SNAP, tungstate and BRz on the expression of NR gene in cucumber explants. Explants were incubated for $24 \mathrm{~h}$ in water (control) or in the presence of the different compounds in concentration as indicated on the figure. Relative expression was determined using the $\Delta \Delta \mathrm{Ct}$ method [37], taking actin gene as a reference. Data were collected from 3 independent biological replicates. Each replicate represents a pool of ten explants. Bars denoted by identical letters did not differ significantly (Duncan's multiple test; $P<0.05$ )

auxin induced adventitious root formation in marigold [30]. The interaction of $\mathrm{NO}$ with other hormones has also been extensively reported $[22,54,55]$. However, the interaction between BR and NO in adventitious root development has hardly been reported. Our results have demonstrated that BR- induced adventitious roots development was inhibited by NO scavenger (c-PTIO) or inhibitors (L-NAME, Tungstate) (Fig. 2). The scavenger and inhibitors of NO not only inhibited the production of endogenous NO, but also inhibited the effect of the exogenous BR. This observation was consistent with the findings of Li et al. [56]. Our results suggested that nitric oxide was involved in the brassinolide-induced adventitious root development in cucumber explants.

In the subsequent experiment, the involvement of endogenous $\mathrm{NO}$ in BR-induced adventitious rooting was further confirmed by the increased activities of NOS-like and NR (Fig. 3). $\mathrm{BR}_{\mathrm{Z}}$ reduced the production of $\mathrm{NO}$ and activities of NOS-like and NR, and its effect was timedependent. Previous studies also showed that methane $\left(\mathrm{CH}_{4}\right)$ triggered the accumulation of $\mathrm{NO}$ in cucumber during adventitious root formation [57]. Ethephon treatments induced the increase of endogenous NO level and significantly improved activities of NOS-like and NR during adventitious rooting [58]. Hydrogen gas induced adventitious rooting by enhancing NO level [22]. Hemin might act to promote $\mathrm{NO}$ accumulation in mediating adventitious root development of cucumber explants [59]. As indicated above, the endogenous accumulation of NO induced by certain exogenous substance could promote adventitious root development. Therefore, our experiment explained the fact that BR promotes the formation of adventitious root by increasing endogenous NO level and improving the activities of NOS-like and NR enzymes. Recently, it was reported that endogenous NO production and activity of NOS-like and NR were inhibited by c-PTIO in the euhalophyte Suaeda salsa [60]. The NO content in tea leaves induced by BR was suppressed when using a NO scavenger [54]. This latest observation was consistent with our results (Fig. 4). We found that exogenous application of BR and SNAP significantly increased endogenous $\mathrm{NO}$ production. However, L-NAME and Tungstate, $\mathrm{BR}_{\mathrm{Z}}$ reversed the positive roles of BR and SNAP in NO accumulation. The induction of NOS-like and NR activities induced by BR were inhibited by L-NAME, Tungstate and $\mathrm{BR}_{\mathrm{Z}}$. In summary, $\mathrm{BR}$ induced the production of endogenous $\mathrm{NO}$ by stimulating the activity of NOS-like and NR enzymes and promoted in the development of adventitious roots in cucumber. In the present study, the endogenous NO production and distribution were visualized in planta by the DAF-FM DA fluorescent probe technology, thus verifying that $B R$ could promote the production and accumulation of endogenous NO during adventitious rooting in cucumber. Moreover, NO inhibitors could reverse the role of $\mathrm{BR}$ in the promotion of endogenous NO, indicating that $\mathrm{NO}$ participated in the process of BR induced adventitious roots in cucumber. Similar studies reported that the production and distribution of endogenous NO were also detected by DAF-2DA, a fluorescent NO indicator dye in Arabidopsis thaliana and in maize [33, 61]. Indeed, it is known that NR activity on 
one hand is regulated by phosphorylation and interaction with 14-3-3 proteins [62,63], on the other hand, the activity of NR is affected by the abundance of NR enzyme in a certain degree. Whereas the abundance of the NR protein is affected by the accumulation of the $N R$ gene transcript. Therefore, we further investigated the regulation of $N R$ gene expression. Our data showed that both BR and SNAP induced the accumulation of the transcript, whereas $\mathrm{BR}_{\mathrm{Z}}$ and Tungstate treatment down-regulated the relative expression level of $N R$ (Fig. 5). These results are similar to that of Zhu et al. [22], who showed that $50 \%$ hydrogen-rich water (HRW) treatment induced adventitious root development and upregulated the expression of $N R$ in cucumber. $\mathrm{Xu}$ et al. [54] also reported that ETH enhanced the relative expression of $N R$ and improved adventitious rooting in cucumber. Taken altogether, the data strongly suggest that BR and SNAP stimulate the activity of NR enzyme, inducing the accumulation of transcripts and probably the corresponding protein, causing the production and accumulation of $\mathrm{NO}$ and promoting, subsequently, adventitious rooting in cucumber. Similarly, in cucumber, Zhu et al. [22] have reported that nitric oxide is required for hydrogen gas-induced adventitious root formation and Pagnussat et al. [29] have reported that nitric oxide and cyclic GMP are involved in the indole acetic acidinduced adventitious rooting process, which suggests that hydrogen gas and indole acetic acid might be the upstream signal molecule of nitric oxide during adventitious rooting process. It will provide us a new research topic about the up-and-downstream relationship of BR, hydrogen gas and indole acetic acid on adventitious root formation.

\section{Conclusions}

In the present study, we demonstrated that both BR and NO played important role during adventitious roots formation in cucumber explants. BR induced adventitious roots formation by up-regulating the relative expression level of $N R$ gene and increasing the activities of NOSlike and NR enzymes, and then improving the endogenous NO level of cucumber explants. In this regard, we argue that $\mathrm{NO}$ is involved in BR-induced adventitious root formation in cucumber explant. In our future research, the interaction mechanisms and signal transduction pathway of BR and $\mathrm{NO}$ will be investigated by molecular and genetics methods.

\section{Methods}

\section{Plant material and growth conditions}

The seeds of cucumber, Cucumis sativus L. var. Xinchun 4, were purchased from Gansu Academy of Agricultural Sciences (Lanzhou, China). The seeds were surfacesterilized in 5\% sodium hypochlorite for $10 \mathrm{~min}$, washed with water, germinated in petri dishes with double- layer filter paper moistened with distilled water. The seeds were put in an electronic growth chamber at $25 \pm 1{ }^{\circ} \mathrm{C}$ for 5 days with a 14-h photoperiod (photosynthetically active radiation $=200 \mu \mathrm{mol} \mathrm{m}^{-2} \mathrm{~s}^{-1}$ ). Primary roots of 5day-old seedlings were excised and then the cucumber explants were maintained under the same conditions of temperature and photoperiod for 5 days under different treatments as indicated below. The analytical grade chemicals used in the study were obtained from Chinese companies. Root number and root length per explant were recorded and analyzed.

\section{Treatments and experimental design}

Cucumber explants were placed in petri dishes (diameter $=9 \mathrm{~cm}$ ) lined with double layer tissue paper and moistened with $60 \mathrm{~mL}$ distilled water as control (control) or $60 \mathrm{~mL}$ of various concentration $(0.2,1,2,4,8 \mu \mathrm{M})$ of Brassinolide (BR, Sigma, USA) and kept at $25 \pm 1{ }^{\circ} \mathrm{C}$. The following chemicals were added with suitable concentration of BR: $50 \mu \mathrm{M}$ SNAP (S-nitroso-N-acerylpenicillamine, Sigma, United Stated), $200 \mu \mathrm{M}$ c-PTIO (2-(4carboxy-2-phenyl)-4,4,5,5-tetramethy limidazoline-1oxyl- 3-oxide, Sigma, United Stated), $20 \mu \mathrm{ML}$ L-NAME (N-nitro-l-arginine methyl ester, Sigma, United Stated) and $100 \mu \mathrm{M}$ Tungstate (Zhongtai Chemical Co.Ltd. Shanghai, China). Moreover, $1.5 \mu \mathrm{M} \mathrm{BR}$ (brassinazole, Sigma, United Stated) was administered to explants. The concentrations of these chemicals were selected based on the results of preliminary experiments (data not shown). The treatments were arranged in a completely randomized design in three replicates. Each experimental unit consisted of ten individual explants from which data were taken. Data are expressed as means \pm standard error (SE).

\section{Determination of root number and length of cucumber explants}

Five days after treatments, the root number of each explant was counted and the root length of each explant was measured with a ruler (accuracy is $0.1 \mathrm{~cm}$ ). Three independent biological replications were done and ten explants of each replication were analyzed. Data were expressed as the average $\pm \mathrm{SE}$.

\section{Determination of the endogenous NO content}

The endogenous NO level was measured as described by Liao et al. [30] using the Griess reagent method. Half of gram $(0.5 \mathrm{~g})$ of hypocotyls (segments $1 \mathrm{~cm}$ long from the base of the hypocotyl) were frozen in liquid nitrogen, then ground with mortar and pestle, and homogenized in $3 \mathrm{~mL}$ of $50 \mathrm{mM}$ ice-cold acetic acid buffer (pH 3.6), containing $4 \%(\mathrm{w} / \mathrm{v})$ zinc diacetate. The homogenates 
were centrifuged at $10,000 \times g$ for $15 \mathrm{~min}$ at $4{ }^{\circ} \mathrm{C}$ and the supernatants were collected.

The pellets were washed using $1.0 \mathrm{~mL}$ of the above extraction buffer and centrifuged. Activated charcoal (0.1 g) was added to the supernatant, which was then filtered and the absorbance was determined at $540 \mathrm{~nm}$. The NO content was calculated by comparison with a standard curve of $\mathrm{NaNO}_{2}$. Measurements were done in 3 independent biological replicates and data represent the average \pm SE.

\section{NOS-like and NR activity determination}

The activities of NR and NOS-like were analyzed by nitrate reductase assay kit and nitric oxide synthase assay kit according to the manufacturer's instructions. The kits were purchased from Nanjing Jiancheng Biological Engineering Co, China. Measurements were done in 3 independent biological replicates and data represent the average \pm SE.

\section{Imaging of endogenous NO by fluorescence microscope}

To follow NO accumulation in planta, hypocotyls $(1 \mathrm{~cm})$ of cucumber explants grown on plates were incubated in the dark for $2 \mathrm{~h}$ in the presence of $20 \mu \mathrm{M}$ DAF-FM DA (4-amino-5-methy- lamino-2, 7-diamino-fluorescein diacetate, sigma) prepared in $50 \mathrm{mM}$ Tris- $\mathrm{HCl}$ ( $\mathrm{pH} 7.5)$. Hypocotyls were extensively washed with distilled water to remove excess of the fluorophore. The hypocotyls under each condition were observed with a fluorescent microscope (Leica 400x, Planapo, German Weizla). Fluorescence intensity was analyzed using ImageJ software and expressed as a percentage of the control. The experiment was repeated three times and ten hypocotyls were observed each time.

\section{Determination of transcript abundance by real-time PCR}

Total RNA was isolated from $100 \mathrm{mg}$ (fresh-weight) of excised cucumber hypocotyls (segments $1 \mathrm{~cm}$ long from the base of the hypocotyl), ground with mortar and pesthe in liquid nitrogen, using plant RNA extraction kit (TaKaRa MiniBEST 9769; TaKaRa Biomedicals, Japan) according to the manufacturer's instructions. Synthesis of cDNA was performed with Prime Script ${ }^{\text {tm }}$ RT reagent Kit (TaKaRa Biomedicals, Japan) starting from $500 \mathrm{ng}$ of total RNA according manual's instructions. The real time quantitative RT-PCR was used to analyze the relative expression of $N R$ genes in hypocotyl of cucumber explant through a SYBR Premix Ex Taq II (Tli RNaseH Plus; TaKaRa Biomedicals, Japan). Actin gene (accession number: DQ641117) was used as an internal control. Gene-specific primers were designed by Primer3plus as followed: for NR (accession number: JQ692875.1), forward 5' -AAACCCTACATCCTTCACTCTCG - 3' and reverse 5' -GGTCCATTGCCATTTCTCTTCT- 3', for actin, forward 5'-CCCATCTATGAGGGTTACGCC-3' and reverse 5' - TGAGAGCATCAGTAAGGTCACGA3 '. The total volume of each reaction was $20 \mu \mathrm{L}$, and contained $10 \mu \mathrm{L}$ SYBR Premix Ex Taq II, $2 \mu \mathrm{L}$ of 10 -fold diluted cDNA and $0.8 \mu \mathrm{L}$ of $10 \mu \mathrm{M}$ forward and reserve primers, to a final volume of $20 \mu \mathrm{L}$ by adding water. Amplification program consisted of one cycle of $95^{\circ} \mathrm{C}$ for $60 \mathrm{~s}, 40$ cycles of $95^{\circ} \mathrm{C}$ for $5 \mathrm{~s}$, and melting analysis at $60^{\circ} \mathrm{C}$ for $20 \mathrm{~s}$, and $95^{\circ} \mathrm{C}$ for $15 \mathrm{~s}$, followed by one cycle of $60{ }^{\circ} \mathrm{C}$ for $60 \mathrm{~s}$, and $95^{\circ} \mathrm{C}$ for $15 \mathrm{~s}$. All qRT-PCR for each gene was performed in three biological replicates, with three technical repeats per experiment. The relative quantification of mRNA levels is based on the method of Livak and Schmittgen [37]. The threshold cycle value (Ct) of actin was subtracted from that of the target gene to obtain a $\Delta \mathrm{Ct}$ value. The $\mathrm{Ct}$ value of the control sample in experiment was subtracted from the $\Delta \mathrm{Ct}$ value to obtain a $\Delta \Delta C t$ value. The expression level relative to the control for each sample was expressed as $2^{-\Delta \Delta C t}$.

\section{Statistical analysis}

Each experiment was repeated three times and the data collected were expressed as mean values \pm standard error (SE). The analysis of variance was performed using SPSS Statistics 17.0 software and treatment means were separated by Duncan's multiple range test $(P<0.05)$.

\section{Abbreviations \\ 24-EBR: 24-epibrassinolide; BL: epibrassinolide; BR: Brassinolide; \\ $\mathrm{BR}_{\mathrm{Z}}$ : Brassinazole; c-PTIO: 2-(4-carboxy-2-phenyl)-4,4,5,5- tetramethylimidazoline-1-oxyl-3-oxide; CTK: cytokinin; $\mathrm{H}_{2} \mathrm{O}_{2}$ : hydrogen peroxide; IAA: indole-3-acetic acid; JA: jasmonic acid; L-NAME: N-nitro-I- arginine methyl ester; NO: Nitric oxide; NOS: NO synthase; NR: Nitrate reductase; SA: salicylic acid; SNAP: S-nitroso-N-acerylpenicillamine}

\section{Acknowledgements}

We thank Dr. Lixiang Cheng (Gansu Provincial Key Laboratory of Arid Land Crop Science, Gansu Agricultural University, Lanzhou 730070, P.R. China) for providing the method support.

Authors' contributions

$Y L$ and JY2 conceived and designed the research. $Y L$ and $W L$ conducted the experiments. YL, YW, XJ, JY1 and ZT analyzed the data and prepared the figures and illustrations. $Y L$ wrote the manuscript. $W L, L H$ and $M D$ read the manuscript and made valuable inputs. All authors read and approved the submission of the manuscript.

\section{Funding}

This research work was financially supported by the National Natural Science Foundation of China (No. 31660584), Agriculture Research System of China (CARS-23-C-07), the Gansu Province Science and Technology Project (17ZD2NA015-03) and the National Key Research and Development Project of China (2018YFD0201205). The funders had no role in the design of the experiments, data collection and analysis, data interpretation and also in writing the manuscript.

Availability of data and materials

The datasets generated during the current study are available from the first author on reasonable request.

Ethics approval and consent to participate Not applicable. 


\section{Consent for publication}

Not applicable.

\section{Competing interests}

The authors declare that they have no competing interests.

\section{Author details}

'College of Horticulture, Gansu Agricultural University, Lanzhou 730070, People's Republic of China. 'Department of Horticulture, FoA, University for Development Studies, P. O. Box TL 1882, Tamale, Ghana.

\section{Received: 26 August 2019 Accepted: 27 February 2020}

\section{Published online: 06 March 2020}

\section{References}

1. Grove MD, Spencer GF, Rohwedder WK, Mandava N, Worley JF, Warthen JD, Steffens GL, Flippen-Anderson JL, Cook JC. Brassinolide, a plant growthpromoting steroid isolated from Brassica napus pollen. Nature. 1979; 281(5728):216-7.

2. Kurepin LV, Bey MA, Back TG, Pharis RP. Structure-function relationships of four stereoisomers of a Brassinolide mimetic on hypocotyl and root elongation of the Brassinosteroid-deficient det2-1 mutant of Arabidopsis. J Plant Growth Regul. 2016;35(1):215-21.

3. Kang Y-Y, Guo S-R, Li J, Duan J-J. Effects of 24-Epibrassinolide on antioxidant system in cucumber seedling roots under hypoxia stress. Agric Sci China. 2007:6(3):281-9.

4. Aghdam MS, Mohammadkhani N. Enhancement of chilling stress tolerance of tomato fruit by postharvest Brassinolide treatment. Food Bioprocess Technol. 2014;7(3):909-14.

5. Tanveer M, Shahzad B, Sharma A, Biju S, Bhardwaj R. 24-Epibrassinolide; an active brassinolide and its role in salt stress tolerance in plants: a review. Plant Physiol Biochem. 2018;130:69-79.

6. Sharma P, Kumar A, Bhardwaj R. Plant steroidal hormone epibrassinolide regulate - heavy metal stress tolerance in Oryza sativa L. by modulating antioxidant defense expression. Environ Exp Bot. 2016;122:1-9.

7. Anjum SA, Wang LC, Farooq M, Hussain M, Xue LL, Zou CM. Brassinolide application improves the drought tolerance in maize through modulation of enzymatic antioxidants and Leaf gas exchange. J Agron Crop Sci. 2011; 197(3):177-85.

8. Yuan L, Zhu S, Shu S, Sun J, Guo S. Regulation of 2,4-epibrassinolide on mineral nutrient uptake and ion distribution in $\mathrm{Ca}\left(\mathrm{NO}_{3}\right)_{2}$ stressed

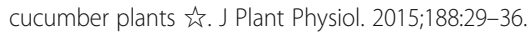

9. Zhao G, Xu H, Zhang P, Su X, Zhao H. Effects of 2,4-epibrassinolide on photosynthesis and Rubisco activase gene expression in Triticum aestivum $\mathrm{L}$. seedlings under a combination of drought and heat stress. Plant Growth Regul. 2017;81(3):377-84

10. Lu Z, Huang M, Ge D-P, Yang Y-H, Cai X-N, Qin P, She J-M. Effect of brassinolide on callus growth and regeneration in Spartina patens (Poaceae). Plant Cell Tissue Organ Cult. 2003;73(1):87-9.

11. Çağ S, Gören-Sağlam N, Çıngıl-Barıs Ç, Kaplan E. The effect of different concentration of Epibrassinolide on chlorophyll, protein and anthocyanin content and peroxidase activity in excised red cabbage ( Brassica Oleraceae L.) cotyledons. Biotechnol Biotechnol Equip. 2007;21(4):422-5.

12. Asami T, Min YK, Nagata N, Yamagishi K, Takatsuto S, Fujioka S, Murofushi N, Yamaguchi I, Yoshida S. Characterization of brassinazole, a triazole-type brassinosteroid biosynthesis inhibitor. Plant Physiol. 2000;123(1):93.

13. Sanz L, Albertos P, Mateos I, Sánchezvicente I, Lechón T, Fernándezmarcos $\mathrm{M}$, Lorenzo O. Nitric oxide (NO) and phytohormones crosstalk during early plant development. J Exp Bot. 2015;66(10):2857.

14. Gouvêa CMCP, Souza JF, Magalhães ACN, Martins IS. NO--releasing substances that induce growth elongation in maize root segments. Plant Growth Regul. 1997;21(3):183-7.

15. Neill SJ, Desikan R, Hancock JT. Nitric oxide signaling in plants. New Phytol New Phytologist. 2003;159(1):11-35

16. Parani M, Rudrabhatla S, Myers R, Weirich H, Smith B, Leaman DW, Goldman SL. Microarray analysis of nitric oxide responsive transcripts in Arabidopsis. Plant Biotechnol J. 2004;2(4):359-66.

17. Delledonne M. NO news is good news for plants. Curr Opin Plant Biol. 2005: 8(4):390-6.

18. Besson-Bard A, Pugin A, Wendehenne D. New insights into nitric oxide signaling in plants. Annu Rev Plant Biol. 2008;59(1):21.
19. Hancock JT, Wilson HR, Neill SJ. Nitric oxide Signalling in plants: John Wiley \& Sons, Itd; 2017.

20. Correa-Aragunde N, Foresi N, Lamattina L. Nitric oxide is a ubiquitous signal for maintaining redox balance in plant cells: regulation of ascorbate peroxidase as a case study. J Exp Bot. 2015;66(10):2913.

21. Astier J, Jeandroz S, Wendehenne D. Nitric oxide synthase in plants: the surprise from algae. Plant Sci. 2018;268:64-6.

22. Zhu Y, Liao W, Wang $M$, Niu L, Xu Q, Jin X. Nitric oxide is required for hydrogen gas-induced adventitious root formation in cucumber. J Plant Physiol. 2016;195:50-8.

23. Tossi V, Lamattina L, Cassia R. Pharmacological and genetical evidence supporting nitric oxide requirement for 2,4-epibrassinolide regulation of root architecture in Arabidopsis thaliana. Plant Signal Behav. 2013;8(7): e24712.

24. Zottini M, Costa A, De MR, Ruzzene M, Carimi F, Lo SF. Salicylic acid activates nitric oxide synthesis in Arabidopsis. J Exp Bot. 2007;58(6):1397.

25. Tun NN, Livaja M, Kieber JJ, Scherer GF. Zeatin-induced nitric oxide (NO) biosynthesis in Arabidopsis thaliana mutants of NO biosynthesis and of twocomponent signaling genes. New Phytol. 2008;178(3):515.

26. Huang $X$, Stettmaier K, Michel C, Hutzler P, Mueller MJ, Durner J. Nitric oxide is induced by wounding and influences jasmonic acid signaling in Arabidopsis thaliana. Planta. 2004;218(6):938.

27. Pagnussat GC, Simontacchi M, Puntarulo S, Lamattina L. Nitric oxide is required for root organogenesis. Plant Physiol. 2002;129(3):954-6.

28. Xiaoting XU, Xin J, Liao W, University GA: Nitric Oxide is Involved in Ethylene-induced Adventitious Root Development in Cucumber Explants. Acta Horticulturae Sinica 2017.

29. Pagnussat GC, Lanteri ML, Lamattina L. Nitric oxide and cyclic GMP are messengers in the indole acetic acid-induced adventitious rooting process. Plant Physiol. 2003;132(3):1241-8.

30. Liao W, Huang G, Yu J, Zhang M, Shi X. Nitric oxide and hydrogen peroxide are involved in indole-3-butyric acid-induced adventitious root development in marigold. J Hortic Sci Biotechnol. 2011;86(2):159-65.

31. Pacurar DI, Perrone I, Bellini C. Auxin is a central player in the hormone cross-talks that control adventitious rooting. Physiol Plant. 2014;151(1):8396.

32. Druege U, Hilo A, Perez-Perez JM, Klopotek Y, Acosta M, Shahinnia F, Zerche S, Franken P, Hajirezaei MR. Molecular and physiological control of adventitious rooting in cuttings: phytohormone action meets resource allocation. Ann Bot. 2019;123(6):929-49.

33. Zhang A, Zhang J, Zhang J, Ye N, Zhang H, Tan M, Jiang M. Nitric oxide mediates brassinosteroid-induced ABA biosynthesis involved in oxidative stress tolerance in maize leaves. Plant Cell Physiol. 2011;52(1):181-92.

34. Deng XG, Zhu T, Zou L, Han XY, Zhou X, Xi DH, Zhang DW, Lin HH. Orchestration of hydrogen peroxide and nitric oxide in brassinosteroidmediated systemic virus resistance in Nicotiana benthamiana. Plant Journal for Cell \& Molecular Biology. 2016;85(4):478.

35. Kwak MS, Kim IH, Kim SK, Han TJ. Effects of Brassinolide with naphthalene acetic acid on the formation of adventitious roots, Trichome-like roots and Calli from cultured tobacco leaf segments, and the expression patterns of CNT103. Journal of Plant Biology. 2009;52(6):511.

36. Karpets YV, Kolupaev YE. Participation of nitric oxide in 24-Epibrassinolideinduced heat resistance of wheat coleoptiles: functional interactions of nitric oxide with reactive oxygen species and Ca ions. Russ J Plant Physiol. 2018;65(2):177-85.

37. Livak KJ, Schmittgen TD. Analysis of relative gene expression data using real-time quantitative PCR and the $2^{-\Delta \Delta C T}$ method. Methods. 2001;25(4): 402-8.

38. Müssig C, Shin GH, Altmann T. Brassinosteroids promote root growth in Arabidopsis. Plant Physiol. 2003;133(3):1261-71.

39. Guan M, Roddick JG. Epibrassinolide-inhibition of development of excised, adventitious and intact roots of tomato (Lycopersicon esculentum): comparison with the effects of steroidal estrogens. Physiol Plant. 1988;74(4): 720-6.

40. Guan M, Roddick JG. Comparison of the effects of epibrassinolide and steroidal estrogens on adventitious root growth and early shoot development in mung bean cuttings. Physiol Plant. 2010;73(3):426-31.

41. Nagata N, Min YK, Nakano T, Asami T, Yoshida S. Treatment of dark-grown Arabidopsis thaliana with a brassinosteroid-biosynthesis inhibitor, brassinazole, induces some characteristics of light-grown plants. Planta. 2000;211(6):781-90. 
42. Ali B, Hayat S, Ahmad A. 28-Homobrassinolide ameliorates the saline stress in chickpea (Cicer arietinum L.). Environ Exp Bot. 2007;59(2):217-23.

43. Ali B, Hayat S, Fariduddin Q, Ahmad A. 24-Epibrassinolide protects against the stress generated by salinity and nickel in Brassica juncea. Chemosphere. 2008;72(9):1387-92.

44. Fariduddin Q, Khanam S, Hasan SA, Ali B, Hayat S, Ahmad A. Effect of 28homobrassinolide on the drought stress-induced changes in photosynthesis and antioxidant system of Brassica juncea L. Acta Physiol Plant. 2009;31(5): 889-97.

45. Engin H, Gokbayrak Z. Effects of 22S, 23S-homobrassinolide and gibberellic acid on formation of double fruits in 'Bing' sweet cherry. Acta Hortic. 2016; 1139:299-302

46. Yuan LB, Peng ZH, Zhi TT, Zho Z, Liu Y, Zhu Q, Xiong XY, Ren CM. Brassinosteroid enhances cytokinin-induced anthocyanin biosynthesis in Arabidopsis seedlings. Biol Plant. 2015;59(1):99-105.

47. Sasse JM. Brassinolide-induced elongation and auxin. Physiol Plant. 2010; 80(3):401-8.

48. Nakamura A, Higuchi K, Goda H, Fujiwara M. T, Sawa S, Koshiba T, Shimada Y, Yoshida S. Brassinolide induces IAA5, IAA19, and DR5, a synthetic Auxin response element in Arabidopsis, implying a cross talk point of Brassinosteroid and Auxin signaling. Plant Physiol. 2003;133(4):1843-53.

49. Vandenbussche F, Callebert P, Zadnikova P, Benkova E, Van Der Straeten D. Brassinosteroid control of shoot gravitropism interacts with ethylene and depends on auxin signaling components. Am J Bot. 2013;100(1):215-25.

50. He Y, Zhang H, Sun Z, Li J, Hong G, Zhu Q, Zhou X, Macfarlane S, Yan F, Chen J. Jasmonic acid-mediated defense suppresses brassinosteroidmediated susceptibility to Rice black streaked dwarf virus infection in rice. New Phytol. 2017;214(1):388.

51. Li X-P, Xu Q-Q, Liao W-B, Ma Z-J, Xu X-T, Wang M, Ren P-J, Niu L-J, Jin X, Zhu Y-C. Hydrogen peroxide is involved in abscisic acid-induced adventitious rooting in cucumber (Cucumis sativus L.) under drought stress. Journal of Plant Biology. 2016;59(5):536-48.

52. Chen Y, Wang M, Hu L, Liao W, Dawuda MM, Li C. Carbon Monoxide Is Involved in Hydrogen Gas-Induced Adventitious Root Development in Cucumber under Simulated Drought Stress. Front Plant Sci. 2017:8:128.

53. Liao W, Xiao H, Zhang M. Role and relationship of nitric oxide and hydrogen peroxide in adventitious root development of marigold. Acta Physiol Plant. 2009:31(6):1279.

54. Xu X-T, Jin X, Liao W-B, Dawuda MM, Li X-P, Wang M, Niu L-J, Ren P-J, Zhu $Y$-C. Nitric oxide is involved in ethylene-induced adventitious root development in cucumber (Cucumis sativus L.) explants. Sci Hortic. 2017;215: 65-71.

55. Jangid KK, Dwivedi P. Physiological and biochemical changes by nitric oxide and brassinosteroid in tomato (Lycopersicon esculentum Mill.) under drought stress. Acta Physiol Plant. 2017;39:3.

56. Li X, Zhang L, Ahammed GJ, Li ZX, Wei JP, Shen C, Yan P, Zhang LP, Han WY. Nitric oxide mediates brassinosteroid-induced flavonoid biosynthesis in Camellia sinensis L. J Plant Physiol. 2017;214:145-51.

57. Qi F, Xiang Z, Kou N, Cui W, Xu D, Wang R, Zhu D, Shen W. Nitric oxide is involved in methane-induced adventitious root formation in cucumber. Physiol Plant. 2017;159(3):366-77.

58. Jin X, Liao DW, Yu JH, Ren MP, Dawuda MM, Wang MM, Niu ML, Li MX, Xu MX: Nitric Oxide Is Involved in Ethylene-Induced Adventitious Rooting in M. Canadian Journal of Plant Science 2017.

59. Xuan W, Xu S, Li M, Han B, Zhang B, Zhang J, Lin Y, Huang J, Shen W, Cui J. Nitric oxide is involved in hemin-induced cucumber adventitious rooting process. J Plant Physiol. 2012;169(11):1032-9.

60. Chen T, Yuan F, Song J, Wang B. Nitric oxide participates in waterlogging tolerance through enhanced adventitious root formation in the euhalophyte Suaeda salsa. Funct Plant Biol. 2016;43:3.

61. Desikan R, Griffiths R, Hancock J, Neill S. A new role for an old enzyme: nitrate Reductase-mediated nitric oxide generation is required for Abscisic acid-induced Stomatal closure in Arabidopsis thaliana. Proc Natl Acad Sci U S A. 2002;99(25):16314-8.

62. Lambeck I, Chi J-C, Krizowski S, Mueller S, Mehlmer N, Teige M, Fischer K, Schwarz G. Kinetic analysis of 14-3-3-inhibited Arabidopsis thaliana nitrate Reductase. Biochemistry. 2010;49(37):8177-86.

63. Athwal GS, Huber JL, Huber SC. Phosphorylated nitrate Reductase and 14-33 proteins. Plant Physiol. 1998;118(3):1041.

\section{Publisher's Note}

Springer Nature remains neutral with regard to jurisdictional claims in published maps and institutional affiliations.
Ready to submit your research? Choose BMC and benefit from:

- fast, convenient online submission

- thorough peer review by experienced researchers in your field

- rapid publication on acceptance

- support for research data, including large and complex data types

- gold Open Access which fosters wider collaboration and increased citations

- maximum visibility for your research: over $100 \mathrm{M}$ website views per year

At BMC, research is always in progress.

Learn more biomedcentral.com/submissions 